\title{
Espacios académicos que aborden la formación de los profesionales que intervienen en el hábitat desde el compromiso con los intereses y necesidades populares
}

\section{Academic spaces that address the training of professionals involved in the habitat from the commitment to popular interests and needs}

\author{
Beatriz H. Pedro* \\ Fecha de recepción: 08-05-2017 - Fecha de aceptación: 16-09-2017 \\ Hábitat y Sociedad (ISSN 2173-125X), n. . 10, noviembre de 2017, pp. 79-98. \\ http://dx.doi.org/10.12795/HabitatySociedad.2017.i10.05
}

\begin{abstract}
We develop the theoretical and practical foundations of the need to construct a new structure of disciplinary formation based on the understanding of the social processes that model the housing environment, questioning the current training parameters and their evaluation criteria and project validation. We propose a training space in real interdisciplinary and multisectoral situations with co-authorial participation with the population. We develop the social approach to design and the project that we support, and show examples of outstanding work.
\end{abstract}

\section{Key words}

Education, Participation, Social transformation, Production and social management of habitat

\section{Resumen}

Desarrollamos los fundamentos teórico-prácticos de la necesidad de construir una nueva estructura de formación disciplinar a partir de la comprensión de los procesos sociales que modelan el entorno habitacional, cuestionando los parámetros actuales de la formación y sus criterios de valoración y validación proyectual. Proponemos un espacio de formación en situaciones reales interdisciplinares y multisectoriales con participación co-autoral con la población. Desarrollamos el enfoque social del diseño y el proyecto que sostenemos, y mostramos ejemplos de trabajo destacados.

\section{Pallabras clave}

Educación; Participación; Transformación social; Producción y gestión social del hábitat

\footnotetext{
* Arquitecta. Profesora Titular Taller Libre de Proyecto Social, Conocimiento Proyectual y Estructuras Resistentes, Universidad de Buenos Aires. C.e.: arqbeatrizp@yahoo.com.ar.
} 


\section{Posicionamiento por otra formación}

Trabajar con la parte de la sociedad donde los problemas son más cuantiosos en número de personas afectadas, más urgentes, hasta podría decirse, incómodamente, más propios; y también más novedosos y desafiantes para los [arquitectos y diseñadores] (Pelli, 2001).

$\mathrm{Al}$ fructífero debate sobre la forma en que se abordan las problemáticas sociales del hábitat en la formación de los arquitectos, nos proponemos aportar algunas reflexiones. Se sostienen en las sistematizaciones que sustentan la propuesta pedagógica del Taller Libre de Proyecto Social (desde ahora TLPS), en el territorio del Área Metropolitana Buenos Aires (desde ahora AMBA), que hemos denominado Enfoque social del diseño y el proyecto.

¿Por qué realizamos estas reflexiones? Porque la escisión entre valoraciones y validaciones hegemónicas de los actuales proyectos y prácticas proyectuales están lejos de aportar a solucionar problemas urbanos que siguen pendientes, en particular los de las poblaciones que viven en condiciones de gran vulnerabilidad.

\section{Contexto social que hace a la necesidad académica}

Acontecimientos nacionales y mundiales ponen cotidianamente en el centro de las miradas las problemáticas urbanas, y la construcción de ciudad desarrollada en las últimas décadas. Son luces que se encienden reclamando reflexión y posicionamiento, en particular a quienes hacemos del proyecto arquitectónico y urbano nuestra profesión.

Esta crisis se enmarca en un contexto internacional en el cual las problemáticas de vivienda y hábitat aparecen como uno de los principales asuntos sociales pendientes de resolución, expresión de la desigual lucha de los pueblos del mundo por tener o mantener un lugar donde vivir y trabajar, ante la ofensiva antipopular de las últimas décadas que ha avasallado logros, conquistas y derechos ganados con años de reclamos y movilización popular.

El derecho a la vivienda adecuada, "a disponer de un lugar donde poder abrigarse si se desea, con espacio, seguridad, iluminación y ventilación adecuadas, infraestructura básica y una situación adecuada en relación con el trabajo y los servicios básicos, todo ello a un costo razonable", y la obligación de garantizar ese derecho a todos, "sean cuales fueren sus ingresos o su acceso a recursos económicos", como establecen las Naciones Unidas, son hoy algunos de los derechos más violados en el mundo.

La construcción del hábitat en manos de bancos, grandes empresas constructoras, desarrolladores urbanos que han construido millones de metros cuadrados, ha modelado el entorno urbano y el territorio al son de sus intereses y necesidades; al boom le siguió el crecimiento de la burbuja inmobiliaria que detonó la crisis económica mundial de los últimos años; sus efectos se recogen en la vida de miles en las ciudades, endeudados, desalojados, viviendo en carpas, en las calles y, hay miles de jóvenes y sus familias sin posibilidades de acceder a la vivienda. 


\section{Sintonía académica y profesional con la producción desigual y excluyente del hábitat socio urbano}

Los contenidos y objetivos de la formación de los arquitectos de nuestro país sintonizaron con los parámetros de este proceso de cambios que se consolidó en los noventa y contribuyeron a naturalizarlos, adaptándose al tipo de desarrollo urbano y territorial que impulsan.

El enfoque dominante de la formación y de los procedimientos, a veces explícito y a veces larvado, que organizan reflexiones y herramientas de intervención, desde enfoques técnico-estéticos, a-sociales, a-contextuales y a-históricos, requieren ser revisados; sirven de sostén, en las ideas y valoraciones, a propuestas y proyectos que impactan al territorio con su intervención, con dimensiones de descripción y análisis unilaterales y limitadas.

Formulaciones teóricas intensamente maceradas en las subjetividades, a través de los sistemas de información, propaganda y educación que extraen su enorme potencia de ser las proposiciones de sectores de las clases dominantes (locales o mundiales). Empalmando con la teoría de la globalización y su proposición de que se abría un nuevo momento en la historia de la humanidad, estos cambios en la producción del mundo físico impactan en los estilos de vida, de comunicación, de consumo.

En el plano ideológico, se alienta una creciente indiferencia por las causas sociales, que lleva a no necesitar conocer ni comprender estos procesos ni los mecanismos que los motorizan, ni hacerse cargo de sus consecuencias (contaminación, residuos, pobreza, exclusión).

Se desarrollan y valoran propuestas que permiten independizarse del contexto, ignorando el conjunto, la totalidad, trabajando con enclaves mono-funcionales cada vez más aislados y autónomos. Esto forma una mirada preparada para "descubrir áreas de oportunidad" en las grandes extensiones "de tierra vacante", sea en la escala territorial (tierras públicas de todo tipo de uso anterior o extensiones de tierra rural que se valoriza por la construcción de las autopistas), en la que se desarrollan proyectos enclaves de barrios cerrados, parques industriales, hipermercados; o en la escala urbana y barrial (hipermercados, torres cerradas, shoppings, museos). Y requiere la valorización del automóvil individual, del cual depende la accesibilidad a estas propuestas.

En nuestro país, casos emblemáticos fueron la implosión del Albergue Warnes para construir un shopping en la Ciudad de Buenos Aires (1992), la construcción de un hipermercado y otro shopping en el exmercado de lanares en el Partido de Avellaneda (1994) y la venta de las tierras del área ferroportuaria para desarrollar un barrio de élite en el principal puerto del país (1994).

En todos ellos la arquitectura fue y es mercantilizada en forma extrema, articulándose con la estrategia comercial de una arquitectura del espectáculo y la simulación, adoptando los parámetros de provisionalidad y obsolescencia simbólica de los productos de consumo. Zonas de la ciudad y sus edificios devienen en objetos comunicacionales y sus programas quedan definidos por criterios y especificaciones del marketing sobre la arquitectura.

La formulación ideológico-académica de estos contenidos estudia la ciudad como partes sin contextualizarlas en los procesos generales; justifica la organización del territorio alrededor de la obtención de beneficio en manchas diferenciales y con provisión inequitativa de infraestructuras y equipamiento (proyecto urbano) que, mejorando las 
relaciones espaciales, permita una reproducción óptima del capital en cualquiera de sus formas de inversión en el territorio.

Estos cambios de programas y escalas de inversión y proyecto impactan en el ejercicio profesional, con nuevos modos de toma de decisiones de proyecto, con nuevas figuras profesionales (desarrolladores) y se establecen nuevos modos de especificaciones del proyecto vinculados a cadenas comerciales globales que "imponen" sus criterios de identidad corporativa; son demandas de un cliente que construye no para sí mismo, sino para vender ese producto.

Manifestando el creciente escepticismo por la transformación positiva del mundo, la "arquitectura" se retira de los aspectos comprometidos del ordenamiento del territorio, la vivienda masiva y el espacio público, los problemas urbanos y el crecimiento de los suburbios; concentrándose en el estrecho y concentrado marco de los grandes encargos corporativos y las residencias privadas acomodadas. Los grandes estudios adoptan una visión ensimismada y auto-referenciada en sus propias obras.

\section{Lo social en la formación disciplinar}

La integración en la formación disciplinar de diseñadores, arquitectos y urbanistas con la temática social requiere tener como referencia y punto de partida la realidad del hábitat con la que se enfrentan nuestras disciplinas. Esta realidad forma parte de las condiciones de vida de nuestro pueblo y en ella se expresan materialmente los intereses contradictorios de la estructura económica y social. ${ }^{1}$

La dinámica poblacional, su distribución en el territorio y el desarrollo urbano se ordenan, modifican y construyen en complejos procesos sociales, económicos, culturales y políticos (de variadas escalas), en constante reconfiguración, en los que participan sectores desiguales que compiten por la apropiación y la determinación concreta que asumen los contenidos de los usos de la ciudad y el territorio con lógicas diferentes, según sus objetivos, necesidades y prioridades.

Consideramos que es necesario comprender esos procesos macro que vive la sociedad en sus diferentes escalas, para poder entender el tipo y carácter de las formas de habitar como consecuencia de los diferentes y desiguales procesos de producción que se realizan desde el Estado, desde el mercado y desde los sectores populares.

En nuestro país, el sostenido aumento -intensificado en la última década- de la población urbana en condiciones de emergencia habitacional y ambiental en los viejos y nuevos espacios del hábitat popular (Cravino, 2008) tiene entre sus causas principales los procesos socioterritoriales de expulsión de población del campo y las formas excluyentes de urbanización del AMBA, que han convertido al territorio nacional en un "plano inclinado" que empuja y aglomera a la población en las ciudades, por lo que se desarrolla un proceso de densificación y consolidación de situaciones con carencias espaciales y ambientales críticas en villas miserias, asentamientos y zonas empobrecidas y relegadas de los centros urbanos.

Los datos muestran que el acceso al suelo urbano para los sectores populares se ha tornado cada vez más dificultoso, pues las villas y asentamientos se constituyen en un lugar posible para habitar la ciudad, y esa situación (Cravino, 2012) se desarrolla dentro de procesos de "gentrificación urbana”, producto de la dinámica de reconfiguración urba-

1 Existe hoy en la Argentina un déficit de más de 3 millones de soluciones habitacionales, que afecta a más de 10 millones de personas. 
na, tanto por procesos largos de mercantilización del hábitat y el habitar como por procesos rápidos de desalojos. ${ }^{2}$

La formación y la práctica profesional dominantes que abordan disciplinas proyectuales están mayoritariamente disociadas de esta realidad. Los planes de estudio y las orientaciones generales les dan la espalda a las necesidades insatisfechas y a los problemas urbanos estructurales, al restringir y canalizar la formación hacia la demanda de un mercado orientado, según los parámetros del negocio inmobiliario y aun de la especulación territorial y edilicia urbana, donde el Estado actúa, a través de regulaciones y políticas, tendiendo a posibilitar la lógica de la ganancia en la producción del hábitat. De ese modo, se puede advertir un fenomenal desarrollo de edificios en altura y de barrios cerrados residenciales - con la consiguiente apropiación de tierras urbanas y rurales- en selectas zonas de la Ciudad de Buenos Aires, el conurbano bonaerense y los principales núcleos urbanos del interior del país.

La falta de respuesta al programa de necesidades de las masas de trabajadores que padecen las mencionadas carencias en materia de tierra y vivienda da impulso a que tomen en sus manos (organizados o por iniciativas familiares o grupales), aunque en condiciones desiguales, la autoproducción y gestión de su hábitat, construyendo espacios habitables, componentes urbanos y viviendas, guiados por el objetivo de satisfacer sus necesidades y derechos.

El arquitecto Fermín Estrella (2003), ${ }^{3}$ desde la propuesta de vivienda y urbanismo social, caracteriza como "pueblos emergentes con necesidades a derechos" a las poblaciones que habitan y producen su hábitat en las múltiples formas que tiene el ambiente popular.

Conceptualizamos este proceso siguiendo los lineamientos que compartimos con otros pensadores y colectivos profesionales y académicos, y que se enmarcan en lo que se denomina teóricamente Producción Social de la Vivienda y el Hábitat, ${ }^{4}$ de ahora en adelante PSH.

Llamamos PSH en sentido amplio a las complejas y heterogéneas prácticas populares que impactan fuertemente en nuestro medio, villas y asentamientos, edificios ocupados, conjuntos habitacionales de distinta escala degradados, barrios populares autoconstruidos, etc. Se expresa en ellos la vida y el esfuerzo de sus habitantes atravesados por décadas de pobreza estructural, informalidad e inestabilidad laboral, desocupación, migración, etcétera.

Este sector mayoritario de productores de vivienda y ciudad constituye un nudo fundamental de la problemática contemporánea de vivienda y hábitat. La inmensa mayoría de las "nuevas viviendas populares", gestionadas por los propios usuarios, sin apoyo técnico ni financiero, muchas veces en forma irregular en lo jurídico y urbanístico y con problemáticas de emergencia ambiental, están sostenidas en tejidos sociales, organizacionales y vinculares que les han dado carnadura, y que es necesario conocer y respetar.

Abordar en forma colectiva y tomando en sus propias manos la lucha por resolver el problema de la vivienda y el hábitat constituye una experiencia reiterada en nuestro país, en distintas épocas y bajo diferentes situaciones político-económicas. Pedro $(2003)^{5}$ estudió en las comunidades barriales del AMBA experiencias que abordaban las problemáticas de hábitat y vivienda, articuladas con las necesidades del trabajo. Se destaca la importante experiencia popular acumulada de formas organizativas, de autogestión y cooperación, y cuestionamiento de respuestas hegemónicas y elaboración de programas de necesidades y propuestas propios.
2 En la CABA hay dos casos emblemáticos del procedimiento de desalojo violento: la ocupación del parque Indoamericano (diciembre de 2010) por miles de familias inquilinas de villas y asentamientos fue desalojada violentamente en menos de una semana con engaños y muertos; y el barrio Papa Francisco, tierra destinada a la reurbanización de la Villa 20, desalojado luego de casi seis meses en que se abandonó a la suerte a las $700 \mathrm{fa}-$ milias, lo que facilitó una zona liberada para justificar el desalojo ante la opinión pública.

3 Vivienda y Urbanismo Social. Recuperado en abril de 2017 de: www.ferminestrella.com.ar.

4 Ortiz, E. "Esencialmente el concepto de producción social del hábitat nos clarifica la comprensión de la existencia de un sistema de producción diferente del modelo dominante, que ha demostrado resultados concretos y sostenibles para los sectores tradicionalmente excluidos del modelo capitalista, donde el suelo y la vivienda se consideran mercancía y no un medio para el adecuado desarrollo de la vida”. Seminario del Movimiento de ocupantes e inquilinos de la CABA. 2007.

5 "Echar raíces", Tesis de maestría de la arquitecta Beatriz Pedro. UNLA. 2006. Inédita. 


\section{Revisar la formación y las valoraciones}

Del estudio crítico de las consecuencias de estas orientaciones, podemos destacar los principales obstáculos académicos y sectoriales de la formación impartida.

Se desarrolla una idea de "profesional global", que subestima y desprecia la necesidad de respuestas propias a las problemáticas nacionales; que desconoce y no se hace cargo de la complejidad del hecho territorial-urbano, la complejidad de los procesos sociales y la complejidad de los procesos ambientales.

Hemos elaborado un cuadro de pares que orientan estos enfoques unilateralizando y antagonizando uno de los aspectos como método de posicionamiento. En el Cuadro 1 indicamos en cada par de conceptos, en cursiva, el aspecto unilateralizado de la concepción formativa hegemónica, que se acentúa en la elaboración de propuestas de proyecto.

\begin{tabular}{|c|c|}
\hline Concepciones de abordaje & Pares de análisis \\
\hline \multirow{6}{*}{$\begin{array}{l}\text { Las problemáticas, los sujetos } \\
\text { y la sociedad }\end{array}$} & complejidad/unilateralidad \\
\hline & complejo/simplificado \\
\hline & integral/unilateral \\
\hline & colectivas/individuales \\
\hline & sujetos de la necesidad/sujetos del negocio \\
\hline & protagonista/espectador \\
\hline \multirow{6}{*}{ El proyecto } & relaciones espaciales y sociales/técnico-estético \\
\hline & contexto urbano/obra aislada \\
\hline & proyecto/proyecto de negocio \\
\hline & innovación apropiada/innovación per se \\
\hline & proyecto proceso/proyecto producto \\
\hline & tecnología apropiada/tecnología universal \\
\hline
\end{tabular}

Caracteriza el entorno material en el que se desarrolla la vida de nuestra sociedad coherentemente, sin hacerse cargo de la totalidad, interpretando y valorando lo construido, clasifica las "preexistencias" como integradas por arquitectura de proposición y arquitectura de producción. Esas clasificaciones y valoraciones, ambas llevadas adelante en el contexto de la producción mercantil de la ciudad y en contextos de encargos de inversión y escala diferentes, incluyen:

\section{La arquitectura de proposición}

Arquitectura como práctica elitista que se valora a sí misma como modelo a seguir, y desarrolla la historia de obras excepcionales y modélicas y sus autores. Son obras realizadas en circunstancias extraordinarias por la excepcionalidad de los condicionantes de obra, de proyecto y de autor. Caracterizadas como realizadas con enfoques cultivados, eruditos, preciosistas, innovadores.

Obras ejemplares, que serán referencia disciplinar, que se proponen sacudir el estado de cosas imperante, porque aportan la reformulación de modelos, tipos, técnicas, procedimientos, programas y 
partidos. Proponen nuevos desafíos, investigan nuevos materiales o soluciones, renuevan las formas. Se corresponde con los edificios institucionales y corporativos, algunos conjuntos de vivienda, parques temáticos, aeropuertos, centros comerciales, o sea, los nuevos programas desarrollados por el proceso socio-urbano descrito. Arquitectura de la abundancia.

\section{La arquitectura de producción}

Arquitectura de satisfacción de los encargos comunes, caracterizados como construcción. Es la que se desarrolla en circunstancias ordinarias (propiedad horizontal, arquitectura comercial e industrial), con el objetivo de dar respuesta a necesidades y propósitos "prácticos", ajustando al máximo las soluciones conocidas con normas, técnicas, economías y procedimientos disponibles. La llevan adelante las pequeñas y medianas empresas constructoras, las empresas inmobiliarias, las instituciones estatales. Arquitectura de la escasez.

Esta caracterización deja fuera a casi el $70 \%$ de lo construido, justamente el hábitat construido y autoconstruido por aquellos sectores sociales que, en su extremo, primero habitan y después construyen, producción mayoritaria en América latina.

Para su comprensión se requiere poner en cuestión los parámetros que sustentan, en la clasificación anterior, una desvalorización de la producción con solo el conocimiento y la ideación popular y la posibilidad de conocer e integrar a los usuarios en los procesos de proyecto.

Víctor Pelli, en la conferencia inaugural del 8. ${ }^{\circ}$ Encuentro de la RED ULACAV, realizó una interesante caracterización de las miradas de estos territorios desde enfoques diferentes, de los que se van a desprender tratamientos y propuestas de intervención diferentes (Cuadro 2).

\begin{tabular}{|l|l|}
\hline Enfoque tradicional & Enfoque propuesto \\
\hline como una apropiación ilegal de territorio & como un agrupamiento de familias en situación de exclusión \\
\hline como un escondrijo de maleantes & $\begin{array}{l}\text { como un barrio autogestionario en evolución y con voca- } \\
\text { ción de integrarse social y jurídicamente a la ciudad }\end{array}$ \\
\hline como un campo de proliferación del clientelismo & $\begin{array}{l}\text { como una resultante lógica de políticas habitacionales in- } \\
\text { adecuadas e insuficientes }\end{array}$ \\
\hline $\begin{array}{l}\text { como un sector urbano impresentable, que desvaloriza } \\
\text { a las propiedades circundantes }\end{array}$ & $\begin{array}{l}\text { como un emergente de una estructura social brutalmente } \\
\text { inequitativa }\end{array}$ \\
\hline $\begin{array}{l}\text { como una ubicación de alto valor de mercado donde co- } \\
\text { rresponde hacer un desarrollo inmobiliario de categoría }\end{array}$ & $\begin{array}{l}\text { como un acto físico y urbano de reivindicación y también } \\
\text { como un acto básico de supervivencia sin alternativas a la vista }\end{array}$ \\
\hline $\begin{array}{l}\text { como un hecho incontenible, y un modo típico de con- } \\
\text { formación de la ciudad latinoamericana }\end{array}$ & \begin{tabular}{l} 
un problema socio-espacial multidimensional y complejo \\
\hline
\end{tabular} \\
\hline
\end{tabular}

La experiencia del Taller Libre de Proyecto Social.

Cuadro 2. Fuente: Elaboración propia.

Propuesta de integración de prácticas universitarias y encuentro de necesidades

La realidad de los planes de estudio de las carreras de arquitectura refleja estas carencias y negaciones de la concepción disciplinar tradicional. Las problemáticas socio-urbanas, su reconocimiento y conocimiento, sus protagonistas y sus necesidades, las condiciones del hábitat y el habitar de las grandes mayorías que habitan y luego construyen, y sus 
derechos no tienen en la formación espacios de estudio y abordaje. De esta realidad surge nuestra experiencia.

Con la profunda crisis y rebelión popular de 2001-2002, irrumpieron a la luz pública abruptamente las postergaciones, carencias y demandas populares. Las tremendas consecuencias sociales de la crisis impactaron en docentes y estudiantes de la Facultad de Arquitectura y Diseño en el ámbito de la Universidad de Buenos Aires, en la que se dictan seis carreras proyectuales. Asimismo, motivaron múltiples iniciativas y acciones sociales, barriales y productivas, para ir al encuentro de los sectores populares, de sus luchas y de sus organizaciones sociales.

El TLPS es una experiencia académica emergente de ese proceso, en el ámbito de la Universidad de Buenos Aires, en la Facultad de Arquitectura y Diseño más grande de América latina en la que se dictan seis carreras proyectuales, que se propuso trabajar críticamente sobre la formación práctica y teórica de los futuros arquitectos y diseñadores, y sistematizar en la formación un enfoque social del diseño y el proyecto para otros modos de ejercicio profesional, integrando formación, investigación y extensión.

\section{Extensión}

En las universidades públicas de la Argentina, desde la reforma universitaria de 1918, el concepto de Extensión Universitaria forma parte hasta el presente de sus estatutos y en su momento implicó combatir la apropiación elitista del conocimiento. Sin embargo, la significación y sentido de este concepto y de las prácticas derivadas de él han tenido y tienen diferentes características: períodos en que se entendía como difusión cultural y democratización del conocimiento; otros como venta de "servicios" educativos o profesionales a empresas o al Estado, o las diversas formas de colaboración en la atención de necesidades y demandas de grupos "vulnerables".

Esas distintas acepciones se corresponden con las políticas dominantes en cada momento: desde la década de 1990, por ejemplo, cuando se impusieron las políticas neoliberales basadas en la reducción del estado, la profundización del proceso de desindustrialización iniciado con la dictadura militar de 1976-1983, la política de privatización y desnacionalización de las empresas e instituciones estatales, y la drástica reducción del presupuesto de la educación universitaria alentando su privatización y la llamada "generación de recursos propios", la Extensión se entendió como una articulación, en diverso grado, de venta de servicios a empresas, asistencia al estado y atención de grupos "vulnerables" focalizados. Y es aún una práctica periférica.

\section{Investigación}

El concepto y las prácticas de investigación comparten este estado de cosas en el que están en pugna los mismos intereses que la sostienen como práctica en sí misma puesta al servicio de intereses sintonizados con estos mismos enfoques y búsquedas comprometidas con transformaciones sociales. Es aún también una práctica limitada y de grupos reducidos.

\section{Formación}

La organización de la estructura curricular de la carrera es emergente de los debates y propuestas de las décadas de los sesenta y setenta, 
aportando importantes avances, organizando la formación, abordando de inicio la práctica proyectual y, alrededor de ella, desarrollando los aspectos específicos de la formación disciplinar. El proceso pedagógico más avanzado se desarrolló en la Universidad de Córdoba de 1970 a 1975, y se llamó Taller Total. Su propuesta organizaba la formación alrededor de Talleres de Proyecto que involucraban todos los aspectos de la formación, articulándolos en horizontal y vertical, y por Proyecto entendían las problemáticas sociales a abordar en la intervención desde la escala arquitectónica a la urbana. Estas experiencias fueron clausuradas con la Dictadura (1976-1983) y desechadas durante décadas.

A estas reflexiones en lo institucional se sumó la búsqueda del encuentro de profundas necesidades:

- de quienes protagonizaban procesos sociales y requerían del aporte de las disciplinas del proyecto; organizaciones y comunidades que tomaban en sus manos - y mantienen hoy— la solución de sus problemas más urgentes - que continúan mayormente irresueltos-, y con ello se volvían protagonistas del proceso de cambio de su realidad: trabajadores autogestionados en las experiencias de fábricas recuperadas; organizaciones barriales asamblearias de cara a la solución de la precariedad habitacional y ambiental; emprendimientos de la economía social; comunidades elevando su voz y reclamando el derecho a la información, a la educación, etc.;

- y de aquellos estudiantes y docentes y profesionales que, con fuerza creciente, cuestionaban el modelo académico y profesional imperante que se vivía en los ámbitos de enseñanza de esas disciplinas.

Recogiendo estas experiencias e integrando estas prácticas universitarias propusimos, construimos y desarrollamos un Taller Libre que llamamos de Proyecto Social que desarrolla su práctica formativa abordando problemáticas socio-habitacionales en situaciones reales.

Lleva 16 años de trabajo formativo con intervenciones proyectuales en el territorio, con el objeto de aportar en un trabajo conjunto a procesos populares en curso. Durante más de una década ha desarrollado más de 50 proyectos que involucraron a cientos de estudiantes y graduados de todo el territorio argentino. ${ }^{6}$

Se basa en la idea de orientar el diseño y la arquitectura y la actividad profesional hacia la atención y solución de las necesidades populares, en el camino de la transformación de las condiciones sociales, para aportar en la búsqueda de soluciones desde el campo específico y, en esa tarea, transformar las propias condiciones socioprofesionales. Asimismo, desarrolla iniciativas que construyen nuevos espacios en la universidad y en la comunidad articulando acciones y saberes profesionales con la acción popular colectiva.

Desde entonces, se han producido cambios en diversos espacios académicos y profesionales que también buscaron sintonizar desde diversas perspectivas con los procesos que conmovieron y conmueven a la sociedad y comparten la disposición de implicarse en la tarea de abordar derechos y necesidades populares.

\section{Enfoque social del diseño y el proyecto}

En este campo de tarea, se requiere una revalorización del papel social del "usuario", reconocer el lugar donde está inserta la persona, su
6 Aunque con distintos formatos organizativos (juntas vecinales, asambleas, cooperativas de producción, empresas recuperadas, organizaciones sociales, etc.), todas las organizaciones, junto con quienes se llevaron a cabo distintos proyectos, comparten el hecho de haber tomado en sus manos el proceso de cambio de su realidad, al abordar problemáticas laborales, productivas, sanitarias, ambientales o habitacionales. Entre otros, se han realizado proyectos de identidad visual, de comunicación sobre soportes gráficos y audiovisuales, diseño de producto y asistencia a la producción, talleres y capacitación para la Cooperativa Renacer, ex Aurora (Ushuaia); Cooperativa 18 de Diciembre y Brukman Confecciones (CABA); Cooperativa Barrio Almafuerte, Villa Palito (San Justo); Cooperativa Mujeres Artesanas de la Villa 31 (CABA); Asociación Civil Mercado Bien Público Bonpland (CABA); Cooperativa Textil de la Federación de Cooperativas René Salamanca (Laferrère); Cooperativa Textil Lucha y Trabajo (CABA). Se han abordado problemáticas ligadas a la vivienda, el equipamiento, la infraestructura y el espacio público en barrios del AMBA y de la CABA: barrio Carlos Mujica (Retiro), barrio Acuba (Lanús), Villa 21 (Soldati), La Loma (Vte. López), barrio 14 de Noviembre (Alte. Brown), barrios La Juanita y María Elena (Laferrère), René Salamanca (González Catán), solo por nombrar algunos. 
medio, su historia, su situación actual, y revalorizar su papel en la producción social del hábitat.

Abordar estos entornos autoproducidos implica comprender que su producción ha requerido la participación activa y protagónica en la gestión, decisión o acción de sus habitantes por iniciativa colectiva o familiar; decisiones y criterios que necesitaron de prácticas de carácter integral, apoyadas en saberes populares sistematizados por la experiencia sin asistencia técnica profesional; con recursos que provienen de esfuerzos de trabajos informales y temporarios; y sus componentes físico-espaciales se desarrollaron en procesos temporales y evolutivos de diferente escala temporal.

La valoración de este proceso requiere ampliar la concepción del proyecto, entendiéndolo como

la acción a través de la cual los colectivos sociales asumen la superación de obstáculos en diversos grados y construyen horizontes, mundos posibles. Al hacerlo, anticipan un horizonte remoto que orienta sus acciones, a fin de construir una nueva realidad más justa, más equitativa y más significativa (Galán, 2011).

Pero bajo el capitalismo, como afirma Harvey (2007),

... solo un sector minoritario de la población, aquellos que toman las decisiones, tiene acceso a procesos [reconocidos por las lógicas proyectuales y las políticas públicas] en los que se practique el diseño y la imaginación, negándosele a la mayoría el juego pleno de la creatividad humana constituyéndose así en una situación profundamente alienante.

Asumir que la humanidad, aprendiendo de sus aciertos y errores, ha construido su sitio de habitación desde mucho tiempo antes de que existieran la arquitectura, la ingeniería y el urbanismo, como conocimientos sistematizados y las políticas públicas de vivienda y hábitat, ha sostenido la propuesta de intervención de Pelli (2006) para el proyecto y construcción de nuevos barrios populares, del involucramiento de la población en la decisión y construcción de su hábitat, mediante la organización comunitaria en diversas modalidades.

Según Pelli, se requiere la

participación activa de los habitantes en el control de las decisiones mayores, y libertad para que puedan hacer su propia contribución al diseño, a la construcción y a la gestión de su solución habitacional en mesas (reales o virtuales) de concertación con todos los actores involucrados.

Esto es aún más pertinente en el caso de la (re)urbanización de villas y asentamientos con el objetivo de su "integración sociourbana", que propone intervenciones físicas que impactan sobre un cuerpo material y social existente por respetar.

Formación para nuevas respuestas y nuevas formas de articulación para acompañar desde los saberes disciplinares procesos participativos, interdisciplinarios, progresivos e intersectoriales

Estos enfoques de abordaje interpelan a los modos y lógicas de proyectar, a las políticas, a los modos burocráticos de gestión, a las norma- 
tivas y legislaciones; y requieren de profesionales capaces de encarar problemáticas sociales en contextos complejos, capaces de trabajar con otros desde una relación de pares complementarios con roles diferentes, de valorar y respetar los saberes populares y su capacidad de elaboración de propuestas (Pedro, 2015).

La valoración de la acción de la comunidad se centra en los fundamentos conceptuales (Max-Neef, 1986) del desarrollo a escala humana y la posibilidad de despliegue del sujeto en su participación, creación, libertad, identidad, protección, entendimiento y subsistencia.

Esto enfatiza la necesidad de trabajar desde una mirada integral las problemáticas del hábitat, desde una concepción interdisciplinaria, disponiéndose al reconocimiento y articulación con movimientos y organizaciones sociales, donde la participación se constituye en un modo de trabajo general que guía las soluciones, abonando la cogestión y la coautoría con los actores sociales.

Esto pone en foco la necesidad de modificar la cultura profesional tradicional tanto en los aspectos de ampliación interdisciplinaria como en las conceptualizaciones y metodologías del proyecto y del proyectar. Planteando una migración del proyecto objeto a-contextual y asocial al proyecto-proceso, situado y comprometido con la perspectiva de los sujetos de la necesidad (Quiroga, 2004).

Las lógicas del proyecto para la intervención en este ámbito de la realidad requieren la conformación de una nueva cultura proyectual que, propiciando el conocimiento profundo de la sociedad en que se habita, se disponga a poner el énfasis en el proceso social (producción social de vivienda y el hábitat), más que en el producto (producción de vivienda social), incorporándose en relación de pares a equipos interdisciplinarios.

Requiere, además, de la valoración de los saberes populares, una nueva valoración de los conocimientos proyectuales, que, según Galán:

Cuentan entre sus competencias, la capacidad de leer los contextos, desarrollando una sensibilidad específica, de aprovechar oportunidades y de enfrentar perturbaciones [...]. Las virtudes que lo califican son su comprensión de los aspectos simbólicos implicados en las prácticas productivas, sus impactos humanos y sociales, su visión sistémica de la tecnología y la posibilidad de actuar en una dinámica productiva, y la utilización de recursos para facilitar procesos cognitivos (Galán, 2011).

Los conocimientos disciplinares y profesionales del proyecto son complementados por las ciencias humanas para poder diseñar un proceso temporal y participativo, junto con un sujeto con nombre y apellido, que autogestiona o cogestiona el mejoramiento de su hábitat residencial.

Esto requiere una nueva relación entre profesionales y comunidad, de "encuentro de saberes y necesidades" (Pedro, TLPS), que supera en el accionar conjunto las limitaciones en el conocimiento de las necesidades (habitacionales) y la determinación de las prioridades, tanto de los habitantes como de los especialistas, lo que fundamenta la fertilidad de su articulación.

Víctor Pelli plantea el arquitecto-actor en reemplazo del arquitecto-autor para referirse a la profesión como parte de la interacción con otras disciplinas y con las familias usuarias. Un proceso de gestión del proceso progresivo de mejoramiento con asistencia técnica interdisciplinaria requiere un proyectista como actor que articule diversos re- 
7 Esta metodología de trabajo se apoya en los fundamentos conceptuales de "arquitectos de la comunidad" elaborados por el arquitecto Rodolfo Livingston; metodología adoptada y desarrollada para el trabajo barrial con organizaciones sociales que sostiene las propuestas de los Encuentros de Arquitectura Comunitaria, tanto nacionales como latinoamericanos, desarrollados desde 2010. En ellos se ha sistematizado la enorme experiencia de esta articulación desarrollada en los últimos 15 años para proponer una nueva modalidad de "servicio de arquitectura y hábitat".

8 A. Jacubovich desarrolla una crítica muy aguda sobre las metodologías de "participación simulada" y explicita nuevas concepciones para una participación protagónica y articulada, en la que sintetiza su experiencia en ciudad Roca Negra, en Lanús. Recuperado de: http:/ / www. cafedelasciudades.com.ar/arquitectura_117.htm, "Construyendo ciudad junto al colectivo organizado".

9 Sobre concepciones de metodología de participación, abordamos estas experiencias en forma crítica, ya que, como sostiene Javier Encina, el término participación viene siendo utilizado para diferentes propósitos, así como por diferentes actores y agentes sociales: como parte de lo discursivo y que no llega a plasmarse en prácticas; como excusa para iniciar cualquier tipo de cambio; como argumento indispensable para asegurar la eficacia de cualquier acción o como forma de control social. Generando una sustitución del contenido de la participación, para convertirse en un discurso que expresa, que es para el pueblo, pero sin el pueblo. 10 Estrella, F., Vivienda productiva, urbanismo social, generación de empleo. "La comunidad es el motor fundamental en la solución de sus propios problemas, y los programas de vivienda social en todos sus aspectos deben organizarse no para, sino con la participación activa y democrática de las familias involucradas".

Para esto la tipificación y simplificación de las diferentes tareas pone a la tecnología, al diseño y al diseñador al servicio de los usuarios y no a estos al servicio de los diseñadores y de la tecnología. El objetivo del diseño participativo es facilitar la toma de decisiones y reducir con ello la posibilidad de errores. cursos de sus saberes con la población involucrada y con el de otros actores públicos y privados. Se trata de una escala de interfase entre el edificio y el territorio o la ciudad, donde se requieren roles de proyectistas y de planificadores. Partiendo de las necesidades y requerimientos "internos" de mejoramiento, se tienen en cuenta las solicitaciones "externas" del entorno. El resultado final será la elaboración de una propuesta de proceso, en la cual el producto es una consecuencia del proceso cogestado y cogestionado, junto con la población involucrada.

La definición de necesidades y satisfactores (organizacionales y materiales) para llevar adelante este emprendimiento plantea la realización de un diagnóstico participativo desde las necesidades "internas" del sector autoproducido; de los criterios de reconfiguración de lo construido (espacial, de habitabilidad, de estructuras, cerramientos e infraestructura); del proceso de autoconstrucción; de los criterios de asignación de las viviendas reconfiguradas y producidas.

Los requerimientos para la elaboración de ese proceso son sintéticamente:

- el reconocimiento de las formas organizativas existentes;

- el conocimiento de la dinámica de tejidos sociales compuestos de familias extensas y con múltiples tramas relacionales que son la base de una producción familiar intergeneracional (De Paula, 2010) y colaborativa;

- la compresión de la forma de los entornos físico-espaciales autoconstruidos como la articulación de recursos formales, materiales y socioculturales relacionados con el sujeto que los habita y su percepción, significación e identificación;

- la elaboración de metodologías que aporten a procesos de toma de decisiones y producción participativos adecuados para diagnosticar, planificar y evaluar las acciones transformadoras de la realidad;

- la elaboración de proyectos-procesos que elaboren un programa de soluciones progresivas y evolutivas.

Siguiendo a De Paula, los desafíos de este complejo proceso de intervención reclaman comprender un entorno social como producto espacio-temporal en procesos sociourbanos complejos que involucran problemáticas ambientales; comprender su producción tecnológica en sentido amplio; comprender un complejo programa de necesidades de diferentes escalas; involucrarse y aportar a sostener un proceso participativo.

\section{Una propuesta de formación experimentada para un plan integral. ${ }^{7}$ Consultorios y talleres de vivienda y hábitat. Algunos ejemplos}

La formación tiene que incorporar el desarrollo y las metodologías participativas (Robirosa, 2002) con protagonismo popular en las decisiones, ${ }^{8}$ para estos procesos de proyecto. ${ }^{9}$

Consideramos necesario practicar la participación para la elaboración conjunta de diagnósticos, propuestas de resolución y proyectos copensados en la producción social del hábitat, aunque esta sea desprolija, inconstante, inacabada, impredecible, e implique conflictos y desorden, ya que favorece el desarrollo de las potencialidades humanas y la apropiación del conocimiento, de lo propuesto y de su realización. ${ }^{10}$ 
El abordaje de las diferentes problemáticas — sostenemos desde el TLPS— ${ }^{11}$ necesita de una etapa de acuerdo con la comunidad respecto de las necesidades y sus satisfactores, que da origen a un trabajo conjunto y a la conformación de un equipo específico, y que permite la definición de tareas concretas en línea con los objetivos e intereses de las partes, para funcionar como articulación de los lineamientos proyectuales y vinculares de los participantes.

La tarea tiene una función estructurante y es bueno aclarar que, entre la primera prefiguración del proyecto y el acuerdo específico de las tareas por asumir y su alcance, pueden mediar cambios y reajustes que impactarán inevitablemente tanto en los objetivos y resultados esperados, y, por ende, en el cronograma inicial, como en la pertinencia de saberes disciplinares específicos o en el grado de complejidad del proyecto, lo que puede requerir también un ajuste en la composición del equipo. Esto exige abordar los proyectos con flexibilidad.

El desarrollo de la experiencia de realizar prácticas formativas organizando y sosteniendo "consultorios de hábitat" en barrios, villas y asentamientos para el abordaje de proyectos de diferente escala, familiar, grupos de familias, manzanas, sectores barriales, nos permitió poner en práctica y conceptualizar metodologías de trabajo que permiten el desarrollo de estos proyectos de procesos coautorales. ${ }^{12}$

\section{Reurbanización en el barrio Padre Carlos Mujica (Villa 31 y 31bis)/ CABA}

Los equipos docentes-estudiantiles del TLPS participaron activamente en diferentes ámbitos de trabajo dentro de la Villa 31 y 31 bis desde 2006, a partir de acuerdos realizados con familias, comedores barriales, ONG sociales, con el cuerpo de delegados, con la Subsecretaría de Tierras de Nación, con el equipo de proyecto de urbanización de la FADU-UBA, ONG profesionales, etc., con el objeto principal de lograr su radicación y (re) urbanización.

En 2009 fue sancionada por la legislatura porteña la Ley N. ${ }^{\circ} 3343$ de urbanización de la villa y organizada la Mesa de Gestión y Planeamiento Multidisciplinaria y Participativa, que definió en un año el proyecto de radicación del barrio, ${ }^{13}$ de este modo, los trabajos de registro, relevamiento, diagnósticos y proyectos de mejoramiento de lo autoconstruido que veníamos realizando cobraron una particular relevancia. Fuimos invitados a participar de la Mesa y a exponer nuestro trabajo. Finalmente, los lineamientos y enfoques que sostiene el Taller para el mejoramiento del tejido existente formaron parte del dictamen de la Mesa. ${ }^{14}$

La propuesta que se integró a la ley sostiene que las acciones tendientes a mejorar la habitabilidad de los barrios deben considerar desde el primer momento tareas de mejoramiento del tejido existente, juntamente con las grandes definiciones y concreciones que hacen a la gran escala del proyecto de urbanización: infraestructura, apertura de calles, definición de espacios públicos, conectividad, relaciones de bordes, etc.

Se trata de acciones con desarrollos temporales ininterrumpidos y prolongados, que necesitan de múltiples miradas para el conocimiento, diagnóstico y propuestas de mejoramiento, y también para acompañamiento y capacitación de los habitantes; y proponemos para llevarlas adelante la instalación de "consultorios de hábitat" en los barrios, integrados por profesionales y estudiantes de arquitectura y diseño, de las ciencias sociales, del derecho, con capacidad de trabajar interdiscipli-
Para incorporar activamente al usuario al mecanismo de decisiones de diseño urbano, funcional, constructivo, de los espacios verdes productivos y las decisiones ambientales, nos valemos de consignas y tareas con el objetivo de consensuar las discusiones o adaptaciones de proyectos elaborados por el grupo de usuarios y el profesional como facilitador. Y hacer frente a los cambios en la etapa de proyecto, de obra, de uso y de ampliación. Los profesionales facilitadores deben ayudar a solucionar cada necesidad familiar personalizando los diseños sin encarecer su costo. 11 Cuaderno de Formación del TLPS, 1, p. 13. Recuperado en abril de 2017 de: http://www.tlps.com.ar/seminario-teorico-practico/bibliografia.

12 Para otros ejemplos de importante significación se puede consultar la ponencia: "En nuestro país, la importante experiencia realizada en el contexto de los Programas de Mejoramiento de Barrios; emergencia habitacional y de urbanización de villas y asentamientos". Otro ejemplo destacado: "Consolidación urbana participativa de Jnane Aztout", Larache, Marruecos. Llevada adelante por equipos académicos liderados por el arquitecto Esteban de Manuel Jerez, de la Universidad de Sevilla.

13 Realizado sobre la base del proyecto anexo a la ley, elaborado por los equipos de Investigación del Instituto de la Espacialidad Humana, dirigidos por el arquitecto Javier Fernández Castro.

14 Recuperado en abril de 2017 de:

http:/ / www.tlps.com.ar/habitat-villa-31-y31-bis-anuario-tlps-2015. 
nariamente y de manera participativa en el abordaje y resolución de la compleja realidad del hábitat popular.

Entendemos que los Consultorios deben insertarse dentro de la estrategia de ejecución del proyecto de urbanización con los siguientes objetivos generales:

- Lograr una comunicación y relación interactiva y de pares consultorio-familia; consultorio-habitantes de la manzana.

- Proponer soluciones de reorganización de los espacios, que respondan a las necesidades y prioridades de cada caso, articulando las necesidades de las viviendas (de las familias que las habitan), con las necesidades de la manzana, con las necesidades de la urbanización.

- Establecer un programa de necesidades y acciones preventivas y correctivas.

- Establecer una normativa de la manzana, del sector y del barrio (articulando especificidades y criterios comunes) para la reorganización y para los futuros crecimientos.

- Proporcionar orientaciones para un adecuado uso de los recursos materiales disponibles y para las soluciones estructurales, de infraestructura y constructivas.

- Acciones de capacitación a los habitantes con el objetivo de formar agentes del hábitat dentro del barrio con capacidad de orientar positivamente a los vecinos en futuras intervenciones.

Proyecto de barrio para la relocalización de familias afectadas por la operatoria ACUMAR ${ }^{15}$

Durante los años 2011 a 2014, un equipo docente-estudiantil del TLPS trabajó con la comisión de afectados por la relocalización de familias a la vera de arroyos dentro de la operatoria ACUMAR, del barrio 14 de Noviembre en Almirante Brown, para desarrollar un proyectoproceso participativo de elaboración del diagnóstico de necesidades, el programa de demandas y el proyecto de soluciones (satisfactores).

Se llevó adelante un proyecto de diseño de barrio, viviendas y equipamientos comunitarios en consultorios y talleres en los que el equipo estudiantil-docente, junto con 70 familias, protagonizó la elaboración coparticipada.

En él se articularon las decisiones de la comisión de afectados respecto de cómo pasar de un asentamiento de viviendas autoconstruidas a un proyecto respetando el arraigo; se elaboraron parámetros de asignación de lotes, superficies, tamaño y proyecto de viviendas, y su localización en el nuevo barrio, atendiendo las necesidades particulares de cada familia articuladas en el colectivo, con sistematización de saberes disciplinares proyectuales, técnicos y de gestión que facilitaron el proyectar.

Se presentó el proyecto a los equipos encargados de la problemática, tanto en el Municipio de Almirante Brown como en el Juzgado de Morón a cargo.

15 Recuperado en abril de 2017 de: http:/ / www.tlps.com.ar/barrio-14-de-noviembre-anuario-tlps-2015.

16 Recuperado en abril de 2017 de: www.slideshare.net/tallerlibredeproyectosocial/proyectodeurbanizacionynuevobarrioenelprediodeLugano.
Reurbanización de la Villa 20. Proyecto de vivienda para el llamado barrio Papa Francisco ${ }^{16}$

En 2014 integramos el equipo que, coordinado por el arquitecto Fermín Estrella, se ofreció a asistir técnicamente a las familias del designado barrio Papa Francisco, en el terreno asignado a la urbaniza- 
ción de la Villa 20 en Lugano y conjuntamente elaboramos un proyecto que se presentó en la legislatura de la ciudad.

Nuestro vínculo inicial con el barrio se dio ese año, en circunstancias complejas, desde el punto de vista socio-económico, estructural y en una situación coyuntural muy delicada.

En febrero de 2014 se produjo la ocupación de parte del terreno donde se ubicaba el cementerio de autos de la PFA y, luego de meses de infructuosas negociaciones con el GCABA, los habitantes del barrio comenzaron a planificar y proyectar su propio barrio en el terreno que por ley se encontraba afectado a la urbanización de la Villa 20.

En esa instancia, un equipo estudiantil-docente del TLPS interviene en acuerdo con los delegados, con la iniciativa de trabajar en ese caso, en pos de la integración práctica y social de las concepciones de vivienda y urbanismo social.

De esa manera, se elaboraron los lineamientos y la propuesta. La solicitud inicial nos planteó el requerimiento de abordar los siguientes temas:

1. Sintetizar la legitimidad del reclamo.

2. Recabar información técnica sobre las posibles formas de saneamiento.

3. Dar fundamento a la propuesta que habían elaborado para ir resolviendo la urbanización, la vivienda y el saneamiento en forma simultánea.

Del diálogo y trabajo compartido en visitas, relevamientos de la situación sociohabitacional, participación de asambleas, reuniones de delegados, de las presentaciones en comisión de vivienda de la legislatura, la Defensoría del Pueblo, el Instituto de la Vivienda de la Ciudad, y los talleres de trabajo, fueron tomando forma los lineamientos y propuestas que presentamos a consideración.

Se diseñó en función de las pautas fundamentales de la Corriente de Urbanismo Social ${ }^{17}$ a la que nos adherimos, que busca aportar propuestas para resolver desde el reconocimiento integral de las necesidades de los afectados, a diferencia de la Corriente del Urbanismo Inmobiliario, cuyo objetivo fundamental es prioritariamente el aumento de la renta urbana, y la provisión de vivienda y servicios a los usuarios que lo puedan adquirir.

El objetivo ha sido proponer un proyecto de urbanización del predio, asignado por Ley N. ${ }^{\circ} 1770 / 05$, para las familias emergentes de la Villa 20 y del esponjamiento para su urbanización, que dé respuesta a la remediación y construcción de las viviendas necesarias, habitables y productivas, ${ }^{18}$ simultáneamente y manteniendo a las familias en el predio. El programa preveía una cantidad de viviendas que incorporaba a las familias asentadas en el predio, a las necesidades de esponjamiento y a las necesidades de vivienda de otras villas de la CABA.

Dentro del proyecto se propone un modelo de organización y gestión para el proceso de construcción, con lo cual se precisan tres instancias:

1. La capacitación y organización de los vecinos en Grupos de Construcción Cooperativos.

2. El diseño de todos los detalles urbanos y arquitectónicos se realizará en un todo de acuerdo con las pautas y patrones del Diseño Social Participativo.
17 Esta corriente llama Urbanismo Social al diseño urbano-territorial-ambiental, cuyas pautas principales primeramente son el mejoramiento de la calidad de vida socioeconómica y cultural respetando los patrones de avecinamiento, valor agregado social urbano, escala humana en la gradación de zonas urbanas, generación de trabajo permanente, provisión de alimentos, uso de energías renovables, tratamiento de desechos en origen, provisión del Equipamiento Social y Servicios Urbanos no prescindibles para las familias involucradas en un todo de acuerdo con las razones e intenciones que requiere la Declaración de Derechos Humanos de NNUU suscrita por la nación argentina.

18 En la Corriente Arquitectónica de Vivienda y Urbanismo Social esto se llama Vivienda Productiva, porque permite producir una gran serie de usos alternativos productivos y recreativos. Para esta Corriente Social del Pensamiento Arquitectónico al servicio de las necesidades populares, cada familia debe tener la posibilidad de ordenar su espacio individualizadamente como quiera y darle a cada $\mathrm{m}^{2}$ el uso que crea necesario en cada momento de su vida familiar. De la misma manera, los espacios abiertos y comunes: espacio verde abierto en planta baja y espacio de azotea tienen las mismas posibilidades y usos socialmente individualizados para cada ocho departamentos. 
3. El diseño constructivo y las pautas, materiales y modalidades de construcción se realizarán en un todo de acuerdo con las pautas y patrones del Diseño Social Participativo.

Estas tres instancias permiten que la construcción sea ejecutada por los propios vecinos asociados en forma cooperativa, y la dirección técnica, supervisión, asesoramiento y seguridad de obra será responsabilidad de los arquitectos y de los profesionales que se designen por vecinos y autoridades para auditar todo el proceso social, organizativo, productivo, constructivo y económico de las obras.

En ese proceso se suman los Consultorios de urbanismo y arquitectura para el diseño definitivo de viviendas y espacios comunes. Así, se propone constituir equipos de arquitectos, estudiantes de arquitectura y trabajadores sociales, con el asesoramiento de abogados y contadores, que trabajen en las unidades de dos edificios para el diseño definitivo de las viviendas con cada familia adjudicataria. Esto se realizará a través de talleres con las familias de cada unidad de dos edificios y con consultorios personalizados por familia.

Como instancia de control, dentro del proyecto presentado en la legislatura, se estipula la creación de la Mesa de Planeamiento Participativo para la Reurbanización del barrio Villa 20, destinada a establecer, elaborar, impulsar la ejecución y controlar el Plan Integral de Reurbanización del barrio.

La Mesa deberá realizar en la villa exposiciones periódicas y debates sobre la evolución del proceso de urbanización de la villa. En estas asambleas los habitantes del barrio tendrán el derecho a pronunciarse y realizar manifestaciones relacionadas con el cumplimiento de la presente ley. Las propuestas deberán ser documentadas debidamente y analizadas expresamente por la Mesa.

\section{Una conclusión}

He constatado que la gente se organiza, enfrenta, resuelve sus problemas y, con el calor de su afecto, funde todas las buenas ideas y las vuelca en el molde de sus necesidades (Fermín Estrella).

La afirmación que antecede vale tanto para los pobladores como para estudiantes y docentes que, junto con miles de profesionales, buscamos practicar nuestra profesión con otro sentido. Nuevas respuestas y nuevas formas de articulación para un proceso participativo, interdisciplinario, progresivo e intersectorial.

Como hemos expuesto,

los conocimientos disciplinares y profesionales del proyecto complementado por las ciencias humanas pueden diseñar un proceso temporal y participativo junto a un sujeto con nombre y apellido, que autogestiona o cogestiona el mejoramiento de su hábitat residencial; con una nueva relación entre profesionales y comunidad, de "encuentro de saberes y necesidades" (Pedro, TLPS) que supere en el accionar conjunto, las limitaciones en el conocimiento de las necesidades (habitacionales) y la determinación de las prioridades tanto de los habitantes, como de los especialistas, fundamentando la fertilidad de su articulación.

El resultado será la elaboración de una propuesta de proceso en el 
cual el producto es una consecuencia del proceso cogestado y cogestionado junto con la población involucrada.

Desde nuestra concepción y experiencia, y en el marco de respeto por viejos y nuevos derechos, ocultados y denegados, apostamos por la formación en situaciones reales abordando la articulación disciplinar y profesional con las organizaciones que los propios pobladores se dan, para construir, elaborar y desarrollar nuevos modos de servicio profesional de arquitectura y hábitat y a otras políticas, que sea capaz de aportar al desarrollo de Programas-proceso de Intervenciones Proyectuales Integrales que permitan un avance significativo en las necesidades de las familias para dar respuesta a una lucha de décadas, aun en este difícil contexto de ciudades desiguales y excluyentes. 
Alberich, T. (2000). Perspectivas de la investigación social. La investigación social participativa. Barcelona: El Viejo Topo.

Barreto, M.A. y Lentini, M. et al. (2015). Hacia una politica integral del hábitat. Aportes para un observatorio de política habitacional en la Argentina. Ed. Café de las Ciudades, Colección Hábitat.

Bellardi, M. y De Paula, A. (1986). Villas miseria: origen, erradicación y respuestas populares. Buenos Aires: Centro Editor de América Latina, Biblioteca Política $\mathrm{N}^{\circ}$ 159.

Blaustein, E. (2001). Prohibido vivir aquí. Una historia de los planes de erradicación de villas de la última Dictadura. Buenos Aires: CMV.

Bourdieu, P. (1990). Espacio social y génesis de las “clases". En Bourdieu, P., pp. 281-310 (Versión original en Actes de La recherche en sciences sociales, 52-53, París, junio de 1984).

- (2000). Efectos de lugar. En Bourdieu, P. La miseria del mundo. Argentina: FCE, pp. 119-124.

Clichevsky, N. (1997). Regularización dominial, ¿̇solución para el hábitat "popular" en un contexto de desarrollo sustentable? En Cuenya, B. y Falú, A. Reestructuración del Estado y política de vivienda en la Argentina. Buenos Aires: Ediciones CBC.

- (2000). Pobreza y acceso al suelo urbano. Algunos interrogantes sobre las políticas de regularización dominial en América Latina. Recuperado el 12 de diciembre de 2014 de: www.aciamericas.coop/doctos/accesoalsuelo2005.pol.

Cravino, M.C. (1999). Las organizaciones villeras en la Capital Federal entre 1989-1996, entre la autonomía y el clientelismo. 1er Congreso Virtual de Antropología y Arqueología. Ciberespacio, octubre de 1998.

- (2006). Las villas de la ciudad. Mercado e informalidad urbana. Buenos Aires: UNGS.

- (2008). Vivir en la Villa. Relatos, trayectorias y estrategias habitacionales. Buenos Aires: ICO-UNGS.

- (2008). Magnitud y crecimiento de las villas y asentamientos en el área metropolitana de Buenos Aires en los últimos 25 años. Actas 14 Encuentro Red Ulacav. Buenos Aires, Argentina.

- (2012). Construyendo barrios. Transformaciones socioterritoriales a partir de los Programas Federales de Vivienda en el Área Metropolitana de Buenos Aires (2004-2009).

Buenos Aires: Ediciones Ciccus y Universidad de General Sarmiento.

Diez, F. (2008). Crisis de autenticidad. Cambios en los modos de producción de la arquitectura argentina. Buenos Aires: Ed. Summa Libros.

De Paula, J. (2008) La habitación como necesidad, el hábitat como satisfactor. 12 mitos a demoler para avanzar en la inserción de la problemática habita- cional en la universidad y una propuesta. En Ulacav, Inserción de las problemáticas habitacionales de áreas urbanas latinoamericanas en la formación docente. Buenos Aires: FADU.

Equipo de Pastoral Villera (1980). La verdad sobre la erradicación de las villas de emergencia en el ámbito de la Capital Federal. Mimeo.

Estrella, F. Vivienda y Urbanismo Social. Recuperado el 6 de abril de 2017 de: www.ferminestrella.com.ar.

Freire, P. (1997). Pedagogía de la autonomía. Buenos Aires: Siglo XXI.

Galán, B. (2011). Diseño, proyecto y desarrollo. Miradas del periodo 2007-2010 en Argentina y Latinoamérica. Buenos Aires: Ed. Wolkowicz.

Harvey, D. (1976) Urbanismo y desigualdad social. México: Ed. Siglo XXI.

Lefebvre, H. (1969). El derecho a la ciudad. Barcelona: Península.

Max-Neef, M. y Elizalde, A. (1993). Desarrollo a escala humana. Barcelona: Icaria.

Merklen, D. (1997). Organización comunitaria y práctica política. Las ocupaciones de tierras en el conurbano de Buenos Aires. Nueva Sociedad, 149, mayo-junio.

Morano, C. y Lorenzetti, A. (1994). Documento conurbano bonaerense. Los distintos espacios sociales y sus personificaciones socioeconómicas. Congreso Nacional de Estudios del Trabajo. Buenos Aires.

Neuhaus, S. (2006). Discurso hegemónico: vaciamiento de la subjetividad. Crisis, descomposición y recomposición de los vínculos. Argentina: Ed. Herramienta.

Núñez, A. (2001). Los unos y los otros en la lucha por la apropiación del espacio. II Congreso virtual de Antropología y Arqueología.

Ortiz Flores, E. (2007). La producción social del hábitat. Mimeo seminario del Movimiento de ocupantes e inquilinos de la CABA. Buenos Aires.

Oszlak, O. (1991). Merecer la ciudad. Los pobres y el derecho al espacio urbano. Buenos Aires: Humanitas-Cedes.

Pampliega de Quiroga, A. (2001). Crisis social y su impacto en la subjetividad. Buenos Aires: Ediciones Cinco.

Pedro, B. (2006). Barrio María Elena. 23 años de experiencia territorial. De la exclusión a la reconstrucción del tejido social de resistencia y potencialidad contrahegemónica. Argentina: Ed. Herramienta.

- (2010). Universidad y práctica social. Reconstruir una práctica y un saber para la transformación. Trabajos y experiencias del Taller Libre de Proyecto Social. Argentina: Ed. Herramienta.

- (2011). Buenos Aires, una ciudad desigual y excluyente en emergencia habitacional. La Marea, 36.

- (2011). Abordaje popular de las necesidades de tierra, hábitat y vivienda en el área metropolitana 
de Buenos Aires, en contextos sociales de urbanización expulsiva y excluyente. Coloquio Injaviu. Colombia.

- (2015). Tesis de investigación: Proyectar con la comunidad. De la Autoría a la coautoría. Del Proyecto al Proyecto Social. Doctorado en Arquitectura. FADU-UBA. Inédito.

Pelli, V. (2001). Revista La Cutuchet. Colegio de arquitectos Pcia. de Buenos Aires.

- (2003). La necesidad como basamento técnico y político de la gestión habitacional. Área, 11 .

- (2006). Habitar, participar, pertenecer. Acceder a la vivienda, incluirse en la sociedad. Argentina: Ed. Nobuko.

Riofrío, G. (1990). El hábitat de los sectores populares urbanos: las visiones de los pobladores. En De marginales a informales. Lima: Desco.

Robirosa, M. (2002). Observaciones sobre organización y estrategias de los sectores populares en vistas a una participación efectiva en proyectos y programas urbanos de desarrollo social y vivienda. Mimeo Seminario FLACAM. Buenos Aires.

Rodríguez, M.C. (2005). Ocupaciones de edificios y politi- cas locales del hábitat en la ciudad de Buenos Aires. Buenos Aires: Ed. El cielo por asalto.

Rodríguez, M.C. (2009). Autogestión, politicas de hábitat y transformación social. Buenos Aires: Espacio Ed.

- (2011). Desigualdad en el acceso al espacio. Territorio, pobreza y concreción de derechos: una relación estratégica. Encrucijadas, 51.

Svampa, M. y Pereyra, S. (2003). Entre la ruta y el barrio. La experiencia de las organizaciones piqueteras. Buenos Aires: Ed. Biblos.

Svampa, M. (2009). Cambio de época. Movimientos sociales y poder político. Buenos Aires: Ed. Siglo XXI y CLACSO.

Szajnberg, D. (2009). Los nuevos asentamientos informales en la Ciudad de Buenos Aires. Buenos Aires: Ediciones FADU, Nobuko.

Tedeschi, S. (2003). Por una política de vivienda respetuosa de los derechos constitucionales y de los Derechos $\mathrm{Hu}$ manos. Buenos Aires: CELS.

Torres, H. (2001). Cambios socioterritoriales en Buenos Aires durante la década del 90. EURE, 27 (80).

Turner, J. (1977). Vivienda. Todo el poder a los usuarios. Madrid: Ed. H. Blume.

Pedro, B. H. (2017). Espacios académicos que aborden la formación de los profesionales que intervienen en el hábitat desde el compromiso con los intereses y necesidades populares. Hábitat y Sociedad, 10, 79-98.

<http://dx.doi.org/10.12795/HabitatySociedad.2017.i10.05> 


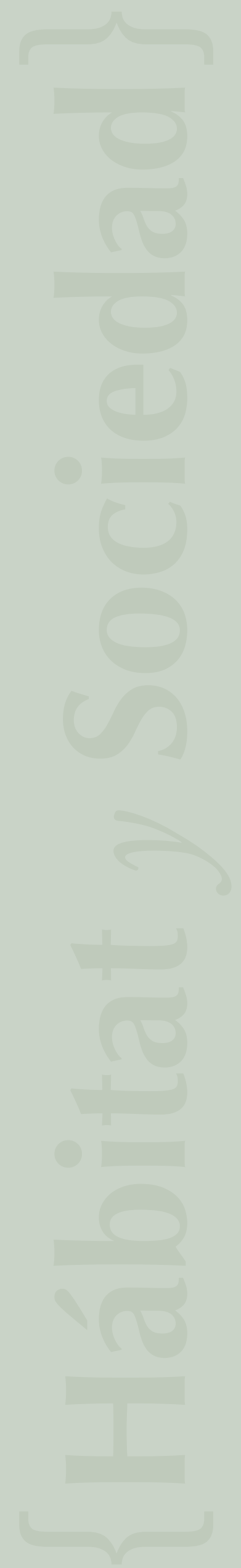

